\title{
Quotienting Share for Dependency Analysis
}

\author{
Andy King ${ }^{1}$, Jan-Georg Smaus ${ }^{1}$, and Pat Hill ${ }^{2}$ \\ 1 University of Kent at Canterbury \\ Canterbury, CT2 7NF, UK \\ amk@ukc.ac.uk, jgs5@ukc.ac.uk \\ 2 School of Computer Studies \\ University of Leeds, Leeds, LS2 9JT, UK \\ hillescs.leeds.ac.uk
}

\begin{abstract}
Def, the domain of definite Boolean functions, expresses (sure) dependencies between the program variables of, say, a constraint program. Share, on the other hand, captures the (possible) variable sharing between the variables of a logic program. The connection between these domains has been explored in the domain comparison and decomposition literature. We develop this link further and show how the meet (as well as the join) of Def can be modelled with efficient (quadratic) operations on Share. Further, we show how by compressing and widening Share and by rescheduling meet operations, we can construct a dependency analysis that is surprisingly fast and precise, and comes with timeand space- performance guarantees. Unlike some other approaches, our analysis can be coded straightforwardly in Prolog.

Keywords. (Constraint) logic programs, abstract interpretation, dataflow analysis, dependency analysis, definite Boolean functions, widening.
\end{abstract}

\section{Introduction}

Many analyses for logic programs, constraint logic programs and deductive databases use Boolean functions to express dependencies between program variables. In groundness analysis $24 \mathrm{I0} Z \mathbf{Z 0} \mathrm{Zb}$, the formula $x \wedge(y \leftarrow z)$ describes a state in which $x$ is definitely ground, and there exists a grounding dependency such that whenever $z$ becomes ground then so does $y$. Other useful properties like definiteness 521 , strictness 19 , and finiteness 6 can be also expressed and inferred with Boolean functions. Different classes of Boolean functions have different degrees of expressiveness. For example, Pos, the class of positive propositional formulae, has the condensing 1 property and is rich enough for goalindependent analysis. Def, the class of definite positive propositional formulae, is less expressive I but has been proposed for goal-dependent analysis of constraint programs 21 .

The objective behind this work was to construct a goal-dependent groundness (and definiteness) analysis for logic (and constraint) programs, that was fast and precise enough to be practical, maintainable and easy to integrate into a Prolog compiler. Binary Decision Diagrams (BDD's) 7 (and their derivatives like ROBDD's) are the popular choice for implementing a dependency analysis 1242026 . These are essentially directed acyclic graphes in which identical sub-graphes are collapsed together. BDD operations require pointer manipulation and dynamic hashing 20 and thus BDD-based Pos analyses are usually 
implemented in C 12426 . Fecht 20 describes a notable exception that is coded in ML. The advantage of using ML is that it is more declarative than $\mathrm{C}$ and therefore easier to maintain. The disadvantage is that it impedes integration into a Prolog compiler 25. The ideal, we believe, is to implement a dependency analyser in ISO Prolog. The problem, then, is essentially one of performance.

Our contribution to solving this problem is as follows: In terms of precision, we provide the first systematic precision experiments that compare Pos and Def for goal-dependent groundness (and definiteness) analysis. We found that Def was as precise as Pos for all our realistic Prolog and CLP $(\mathcal{R})$ benchmarks. We build on this and demonstrate how Def can be implemented efficiently and coded succinctly in Prolog. Our starting point is the work of Cortesi et al 1516 that shows that Share, which is a domain whose elements are sets of sets of variables, can be used to encode Def. We develop this to show:

- how the meet and join operations of Def can be computed straightforwardly based on this encoding, without the closure operation of Share 22 that has a worst-case exponential complexity;

- how an operation (that we call compression) aids fixpoint detection;

- how meet operations can be rescheduled to improve efficiency;

- how widening can be applied to ensure that both the time-complexity of the analysis (the number of iterations) and the space-complexity (the number of sets of variables), grows linearly in the size of the program;

- that the speed of our analysis compares surprisingly well against state-ofthe-art BDD-based Pos analysers 420 .

The rest of the paper is structured as follows. Section $\boldsymbol{Z}$ surveys the necessary preliminaries. Section 3 recalls the relation between Share and Def and is included so that the paper is self-contained. Section 4 shows how the meet and join operations of Def can be computed efficiently using a Share based representation. Section 5 introduces compression and meet scheduling whereas Section 6 discusses widening. Section $\mathbf{\nabla}$ describes the implementation. Section $\mathbf{\nabla}$ reviews the related work, and finally Section 9 presents our conclusions.

\section{Preliminaries}

In this section, we introduce some notation and recall the definitions of Boolean functions and the domain Share. For a set $S,|S|$ denotes the cardinality and $\wp(S)$ the powerset of $S$. Var denotes a denumerable set (universe) of variables and $X \subset \operatorname{Var}$ denotes a finite set of variables; the set of variables occurring in a syntactic object $o$ is denoted by $\operatorname{var}(o)$; the set of all idempotent substitutions is denoted by $S u b$; and Bool is defined to be $\{$ true, false $\}$.

If $(S, \preceq)$ is a poset with top and bottom elements, and a meet sqcap and join $\sqcup$, then the 4 -tuple $\langle S, \preceq, \sqcap, \sqcup\rangle$ denotes the corresponding lattice. A map $g: L \rightarrow K$, where $L$ and $K$ are lattices, is a homomorphism iff $g$ is join-preserving and meet-preserving, that is, $g(a \sqcup b)=g(a) \sqcup g(b)$ and $g(a \sqcap b)=g(a) \sqcap g(b)$ for all $a, b \in L$. An isomorphism is a bijective homomorphism. 


\subsection{Boolean Functions}

A Boolean function is a function $f:$ Bool $^{n} \rightarrow$ Bool where $n \geq 0$. A Boolean function can be represented by a propositional formula over $X$ where $|X|=n$. The set of propositional formulae over $X$ is denoted by $B o o l_{X}$. We use Boolean functions and propositional formulae interchangeably without worrying about the distinction $\mathrm{II}$. We follow the convention of identifying a truth assignment with the set of variables that it maps to true.

Definition $1\left(\operatorname{model}_{X}\right)$. The (bijective) map $\operatorname{model}_{X}: \operatorname{Bool}_{X} \rightarrow \wp(\wp(X))$ is defined by: $\operatorname{model}_{X}(f)=\{M \subseteq X \mid(\wedge M) \wedge(\neg \vee X \backslash M) \models f\}$.

Example 1. If $X=\{x, y\}$, then the function $\{\langle$ true, true $\rangle \mapsto$ true, $\langle$ true, false $\rangle$ $\mapsto$ false,$\langle$ false, true $\rangle \mapsto$ false, $\langle$ false, false $\rangle \mapsto$ false $\}$ can be represented by $x \wedge y$. Also model $_{X}(x \wedge y)=\{\{x, y\}\}$ and model $_{X}(x \vee y)=\{\{x\},\{y\},\{x, y\}\} . \mathbf{I}$

Definition $2\left(\operatorname{Pos}_{X}, \operatorname{Def}_{X}, \operatorname{Mon}_{X}\right)$. $\operatorname{Pos}_{X}$ is the set of positive Boolean functions over $X$. A function $f$ is positive iff $X \in \operatorname{model}_{X}(f)$. $\operatorname{Def}_{X}$ is the set of positive functions over $X$ that are definite. A function $f$ is definite iff $M \cap$ $M^{\prime} \in \operatorname{model}_{X}(f)$ for all $M, M^{\prime} \in \operatorname{model}_{X}(f)$. Mon $X$ is the set of monotonic Boolean functions over $X$. A function $f$ is monotonic iff $M \in$ model $_{X}(f)$ implies $M^{\prime} \in \operatorname{model}_{X}(f)$ for all $M^{\prime}$ such that $M \subseteq M^{\prime} \subseteq X$.

Note that $\operatorname{Def}_{X} \subseteq \operatorname{Pos}_{X}$ and $\operatorname{Mon}_{X} \nsubseteq \operatorname{Pos}_{X}$. It is possible to show that each $f \in D e f_{X}$ is equivalent to a conjunction of definite (propositional) clauses, that is, $f=\wedge_{i=1}^{n}\left(y_{i} \leftarrow \wedge Y_{i}\right)$ 18.

Example 2. Suppose $X=\{x, y, z\}$ and consider the following table, which states, for some Boolean functions, whether they are in $\operatorname{Def}_{X}, \operatorname{Pos}_{X}$ or $M o n_{X}$, and also gives model $_{X}$.

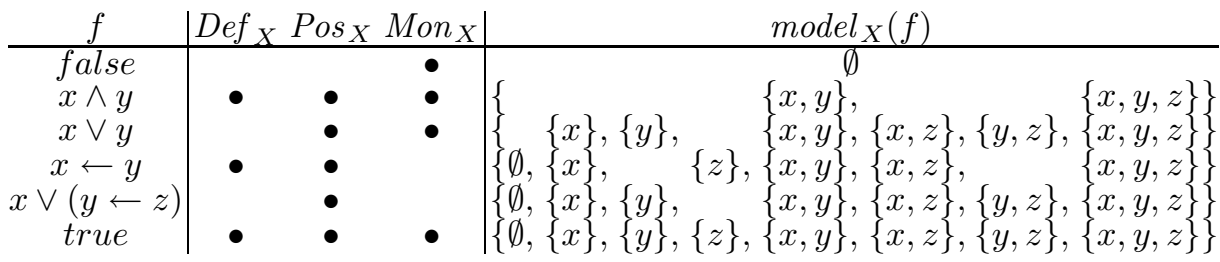

Note, in particular, that $x \vee y$ is not in $D e f_{X}$ (since its set of models is not closed under intersection) and that false is neither in $\operatorname{Pos}_{X}$ nor Def $f_{X}$.

Defining $f_{1} \dot{\vee} f_{2}=\wedge\left\{f \in D e f_{X} \mid f_{1} \models f \wedge f_{2}=f\right\}$, the 4-tuple $\left\langle D e f_{X}, \models, \wedge, \dot{V}\right\rangle$ is a finite lattice 1 , where true is the top element and $\wedge X$ is the bottom element. Existential quantification is defined by Schröder's Elimination Principle, that is,

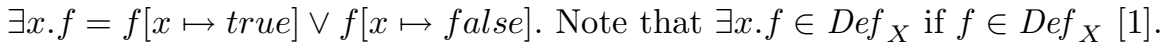

Example 3. If $X=\{x, y\}$ then $x \dot{\vee}(x \leftrightarrow y)=\wedge\{(x \leftarrow y)$, true $\}=(x \leftarrow y)$, as can be seen in the Hasse diagram for $\operatorname{Def}_{X}$ (Fig. II . Note also that $x \dot{\vee} y=$ $\wedge\{$ true $\}=$ true $\neq(x \vee y)$. 

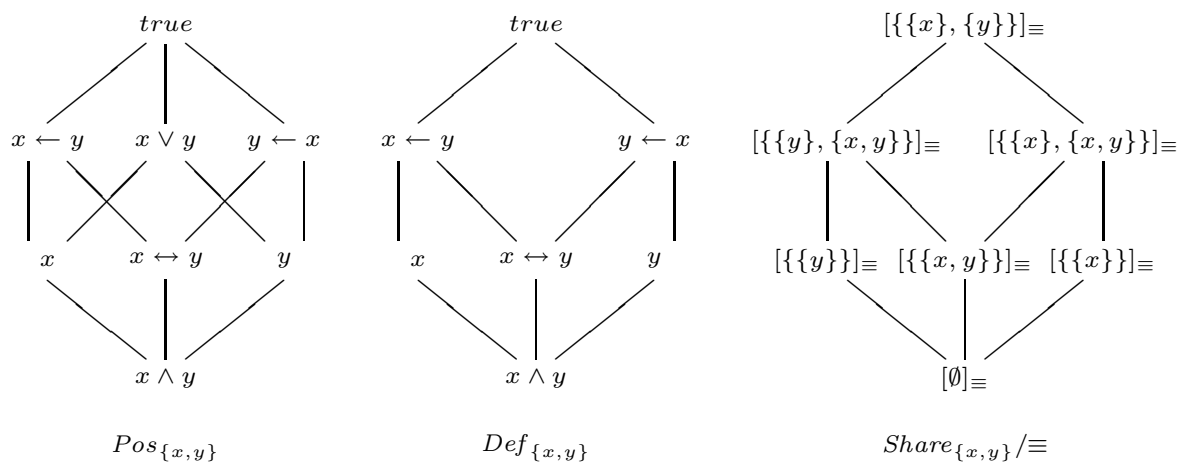

Fig. 1. Hasse diagrams

The maximum number of iterations of a fixpoint analysis relates to the length of the longest ascending chain in the underlying domain. For Pos ${ }_{X}$, it is well-known that the longest chain has length $2^{n}-1$ where $|X|=n$. It is less well-known that the same holds for $\operatorname{Def}_{X}$.

Proposition 1. Let $|X|=n$. Let $f_{1} \models f_{2} \ldots \models f_{k}$ be a maximal strictly ascending chain where $f_{i} \in D e f_{X}$ for all $i \in\{1, \ldots, k\}$. Then $k=2^{n}$.

\subsection{Sharing Abstractions}

For completeness, we introduce the basic ideas behind the Share domain 22. This domain traces the possible variable sharing behaviour of a logic program. Two variables share if they are bound to terms that contain a common variable.

Definition 3 ( Share $\left._{X}\right)$. Share $_{X}=\wp(\wp(X) \backslash\{\emptyset\})$.

Thus we have the finite lattice $\left\langle\right.$ Share $\left._{X}, \subseteq, \cap, \cup\right\rangle$. The top element is $\wp(X) \backslash\{\emptyset\}$ and the bottom element is $\emptyset$.

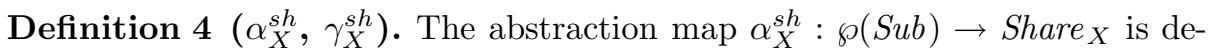
fined as $\alpha_{X}^{s h}(\Theta)=\{\operatorname{occ}(\theta, v) \cap X \mid \theta \in \Theta \wedge v \in \operatorname{Var}\} \backslash\{\emptyset\}$ where $\operatorname{occ}(\theta, v)=\{x \in$ $\operatorname{Var} \mid v \in \operatorname{var}(\theta(x))\}$. The concretisation map $\gamma_{X}^{s h}:$ Share $_{X} \rightarrow \wp(S u b)$ is defined as $\gamma_{X}^{s h}(S)=\left\{\theta \in S u b \mid \alpha_{X}^{s h}(\{\theta\}) \subseteq S\right\}$.

To streamline the theory and reduce the size of abstractions, the empty set is never included in a share set. However there is some loss of information. That is, if every element of $\Theta$ maps every element of $X$ to a ground term then $\alpha_{X}^{s h}(\Theta)=\{\emptyset\} \backslash\{\emptyset\}=\emptyset=\alpha_{X}^{s h}(\emptyset)$. Thus $\alpha_{X}^{s h}$ (and hence $\gamma_{X}^{s h}$ ) cannot distinguish between a set of ground substitutions and the empty set. In practice, the empty set only arises when a computation fails and this would normally be flagged elsewhere in the analyser 9 . 
Example 4. Let $X=\{x, y, z\}$ and consider abstracting (a) $x=f(y, z)$ (b) where at program point (a), no variable in $X$ is ground or shares with any other element of $X$. The bindings on $X$, for example, could be $\theta_{a}$ or $\vartheta_{a}$ as given below. Then the bindings at (b) would be $\theta_{b}$ or $\vartheta_{b}$, respectively.

$$
\begin{array}{ll}
\theta_{a}=\{y \mapsto g(u), z \mapsto v\} & \theta_{b}=\{x \mapsto f(g(u), v), y \mapsto g(u), z \mapsto v\} \\
\vartheta_{a}=\{x \mapsto f(u, u)\} & \vartheta_{b}=\{x \mapsto f(y, y), z \mapsto y\}
\end{array}
$$

The abstraction $S_{a}=\{\{x\},\{y\},\{z\}\}$ describes $\theta_{a}$, that is $\theta_{a} \in \gamma_{X}^{s h}\left(S_{a}\right)$, since $\operatorname{occ}\left(\theta_{a}, x\right)=\{x\}, \operatorname{occ}\left(\theta_{a}, u\right)=\{u, y\}, \operatorname{occ}\left(\theta_{a}, v\right)=\{v, z\}$ and $\operatorname{occ}\left(\theta_{a}, y\right)=$ $\operatorname{occ}\left(\theta_{a}, z\right)=\emptyset$. Similarly $\vartheta_{a} \in \gamma_{X}^{s h}\left(S_{a}\right)$. The abstract unification operation of Jacobs and Langen 22 will compute the abstraction $S_{b}=\{\{x, y\},\{x, z\},\{x, y, z\}\}$ for the program point (b). A safety result of Jacobs and Langen 22 asserts that $\theta_{b}, \vartheta_{b} \in \gamma_{X}^{s h}\left(S_{b}\right)$. Indeed, we see that $\theta_{b} \in \gamma_{X}^{s h}\left(S_{b}\right)$ since occ $\left(\theta_{b}, u\right)=\{u, x, y\}$, $o c c\left(\theta_{b}, v\right)=\{v, x, z\}$, and $\operatorname{occ}\left(\theta_{b}, x\right)=\operatorname{occ}\left(\theta_{b}, y\right)=\operatorname{occ}\left(\theta_{b}, z\right)=\emptyset$. The reader is encouraged to verify that $\vartheta_{b} \in \gamma_{X}^{s h}\left(S_{b}\right)$.

\section{Quotienting Share $_{\boldsymbol{X}}$ to obtain $\operatorname{Def}_{\boldsymbol{X}}$}

In this section we construct a homomorphism from Share $_{X}$ to Def $_{X}$. We recall the well-known connection between Share $_{X}$ and Def $_{X} 13141516$. For the elements of Share $_{X}$, we define an abstraction $\alpha_{X}$ which interprets a sharing abstraction as representing a set of models and hence a Boolean function.

Definition $5\left(\alpha_{X}\right)$. The (abstraction) map $\alpha_{X}:$ Share $_{X} \rightarrow$ Def $_{X}$ is defined as follows: $\alpha_{X}(S)=\operatorname{model}_{X}^{-1}\left(\left\{X \backslash\left(\cup S^{\prime}\right) \mid S^{\prime} \subseteq S\right\}\right)$.

The definition of $\alpha_{X}$ is essentially that of $\alpha$ of Cortesi et al 14 Section 8.4], adapted to our definition of Share ${ }_{X} \cdot \alpha_{X}$ is well-defined, that is, $\alpha_{X}(S) \in \operatorname{Def}_{X}$ for all $S \in$ Share $_{X}$. First, since $X \in$ model $_{X}\left(\alpha_{X}(S)\right)$, it follows that $\alpha_{X}(S) \in$ Pos $_{X}$. Secondly, if $M_{1}, M_{2} \in \operatorname{model}_{X}\left(\alpha_{X}(S)\right)$ then $M_{i}=X \backslash\left(\cup S_{i}\right)$ where $S_{i} \subseteq S$ $(i=1,2)$. Clearly $S_{1} \cup S_{2} \subseteq S$. As $M_{1} \cap M_{2}=X \backslash\left(\cup\left(S_{1} \cup S_{2}\right)\right)$, it follows that $M_{1} \cap M_{2} \in \operatorname{model}_{X}\left(\alpha_{X}(S)\right)$.

Lemma 1. $\alpha_{X}$ is surjective.

However, $\alpha_{X}$ is not injective, and thus it is a strict abstraction of Share ${ }_{X}$. As an example, consider $X=\{x, y\}, S_{1}=\{\{x\},\{y\}\}$ and $S_{2}=S_{1} \cup\{\{x, y\}\}$. Then $\alpha_{X}\left(S_{1}\right)=$ model $_{X}^{-1}(\{\emptyset,\{x\},\{y\},\{x, y\}\})=\alpha_{X}\left(S_{2}\right)$ but $S_{1} \neq S_{2}$.

Example 5. Let $X=\{x, y, z\}$ and $S=\left\{G_{1}, G_{2}, G_{3}\right\}$ where $G_{1}=\{x\}, G_{2}=$ $\{y, z\}$ and $G_{3}=\{z\}$. The table illustrates how $\alpha_{X}(S)$ can be computed by enumerating $\cup S^{\prime}$ and $X \backslash\left(\cup S^{\prime}\right)$ for all $S^{\prime} \subseteq S$.

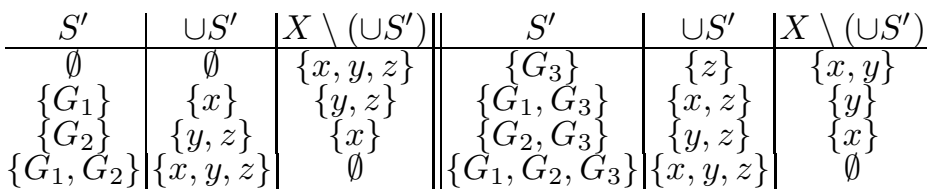

Thus $\alpha_{X}(S)=$ model $_{X}{ }^{-1}(\{\emptyset,\{x\},\{y\},\{x, y\},\{y, z\},\{x, y, z\}\})=(y \leftarrow z)$. The reader is encouraged to verify that $\alpha_{X}(\emptyset)=\wedge X$ and $\alpha_{X}(\{\{x\} \mid x \in X\})=$ true. I 
It is perhaps easier to interpret an abstraction of Share $_{X}$ as definite Boolean functions by using the $\mathcal{C}:$ Share $_{X} \rightarrow$ Def $_{X}$ abstraction map of Cortesi et al 1516 . $\mathcal{C}$ can be expressed particularly succinctly using the auxiliary operation rel which, given a set of variables $G$ and an $S \in$ Share $_{X}$, selects the subset of $S$ which is relevant to the variables of $G$.

Definition $6(\mathrm{rel})$. The map rel $: \wp(X) \times$ Share $_{X} \rightarrow$ Share $_{X}$ is defined by:

$$
\operatorname{rel}(Y, S)=\{G \in S \mid G \cap Y \neq \emptyset\}
$$

Definition 7. The map $\mathcal{C}:$ Share $_{X} \rightarrow \operatorname{Def}_{X}$ is defined by $\mathcal{C}(S)=\wedge F$ where

$$
F=\{y \leftarrow \wedge Y \mid y \in X \wedge Y \subseteq X \backslash\{y\} \wedge \operatorname{rel}(\{y\}, S) \subseteq \operatorname{rel}(Y, S)\} .
$$

$F$ is defined with $Y \subseteq X \backslash\{y\}$ rather than $Y \subseteq X$ to keep its size manageable.

Example 6. Consider again Example 5 The set of $Y \subseteq X \backslash\{x\}$ such that $\operatorname{rel}(\{x\}, S) \subseteq \operatorname{rel}(Y, S)$ is $\{\{x\},\{x, y\},\{x, z\},\{x, y, z\}\}$. Likewise, set of $Y \subseteq X \backslash$ $\{y\}$ such that $\operatorname{rel}(\{y\}, S) \subseteq \operatorname{rel}(Y, S)$ is $\{\{y\},\{z\},\{x, z\},\{x, y\},\{y, z\},\{x, y, z\}\}$. Finally, the set of $Y \subseteq X \backslash\{z\}$ such that $\operatorname{rel}(\{z\}, S) \subseteq \operatorname{rel}(Y, S)$ is $\{\{z\}$, $\{x, z\},\{y, z\},\{x, y, z\}\}$. Thus $\mathcal{C}(S)=(y \leftarrow z)$.

The following proposition asserts the equivalence of $\mathcal{C}$ and $\alpha_{X}$. It is proven by Cortesi et al 14, albeit for slightly different definitions. Modifying their proof to our definitions is straightforward.

Proposition 2. $\mathcal{C}=\alpha_{X}$.

By defining $S \equiv S^{\prime}$ iff $\alpha_{X}(S)=\alpha_{X}\left(S^{\prime}\right), \alpha_{X}$ induces an equivalence relation on Share $_{X}$ which quotients Share $_{X}$. Using the closure under union operation of Jacobs and Langen $\%$, we obtain a useful lemma about these equivalence classes.

Definition 8. Let $S \in$ Share $_{X}$. Then the closure under union $S^{\star}$ of $S$ is defined by: $S^{\star}=\left\{\cup S^{\prime} \mid S^{\prime} \subseteq S\right\} \backslash\{\emptyset\}$.

Note that closure under union is exponential.

Lemma 2. Let $S_{1}, S_{2} \in$ Share $_{X}$. Then $S_{1}^{\star} \equiv S_{1}$ and $S_{1} \equiv S_{2}$ iff $S_{1}^{\star}=S_{2}^{\star}$

We lift $\alpha_{X}$ to $\alpha_{X}:$ Share $_{X} / \equiv \rightarrow$ Def $_{X}$ by defining $\alpha_{X}\left([S]_{\equiv}\right)=\alpha_{X}(S)$. Since $\alpha_{X}:$ Share $_{X} \rightarrow$ Def $_{X}$ is surjective it follows that $\alpha_{X}:$ Share $_{X} / \equiv \rightarrow$ Def $_{X}$ is bijective. We now define, for the the operations $=, \dot{\vee}$ and $\wedge$ on $D e f_{X}$, analogous operations $\sqsubseteq, \sqcup$ and $\sqcap$ on Share $_{X} / \equiv$.

Definition 9 (巨, $\sqcup, \sqcap)$.

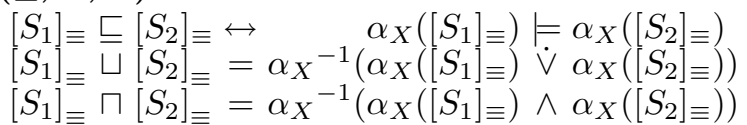

Proposition 3. $\left\langle\right.$ Share $\left._{X} / \equiv, \sqsubseteq, \sqcap, \sqcup\right\rangle$ is a finite lattice.

It follows by construction that $\alpha_{X}$ is an isomorphism. For the dyadic case, the isomorphism is illustrated in Fig. II 


\section{Computing the join and meet within Share}

In this section we show how the meet (as well as the join) of $D e f_{X}$ can be computed with Share $_{X} / \equiv$ via the isomorphism. It is not obvious from the definition of $\dot{\vee}$ how $f_{1} \dot{\vee} f_{2}$ is computed, and it turns out that $f_{1}$ and $f_{2}$ must be put into (orthogonal) reduced monotonic body form 1 . In contrast, it is well-known 1516 that with the Share representation, join basically reduces to set union.

Proposition 4. $\left[S_{1}\right]_{\equiv} \sqcup\left[S_{2}\right]_{\equiv}=\left[S_{1} \cup S_{2}\right]_{\equiv}$

Example 7. Consider calculating $\left[S_{1}\right]_{\equiv} \sqcup\left[S_{2}\right]_{\equiv}$ where $X=\{w, x, y, z\}, S_{1}=$ $\{\{w, x, y\},\{x, y\},\{y\},\{z\}\}$ and $S_{2}=\{\{w, z\},\{x\},\{y\},\{z\}\}$. Note that $\alpha_{X}\left(S_{1}\right)=$ $(w \leftarrow x) \wedge(x \leftarrow y)$ and $\alpha_{X}\left(S_{2}\right)=w \leftarrow z$. Then $\alpha_{X}\left(S_{1} \cup S_{2}\right)=\alpha_{X}(\{\{w, x, y\}$, $\{w, z\},\{x\},\{x, y\},\{y\},\{z\}\})=(w \leftarrow(x \wedge z)) \wedge(w \leftarrow(y \wedge z))$ as required.

The challenge is in defining a computationally efficient meet. This is defined in terms of a map iff which, in turn, is defined in terms of the binary-union operation of Jacobs and Langen $\%$. We follow Cortesi et al 16 and denote binary union as $\otimes$.

Definition 10 (binary-union, $\otimes$ ). The map $\otimes:$ Share $_{X}^{2} \rightarrow$ Share $_{X}$ is defined by: $S_{1} \otimes S_{2}=\left\{G_{1} \cup G_{2} \mid G_{1} \in S_{1} \wedge G_{2} \in S_{2}\right\}$.

The if and iff maps defined below are similar to the classical abstract unification operation of Jacobs and Langen 22. Their interpretation, however, is that given variable sets $Y_{1}$ and $Y_{2}$ and an abstraction $S$ such that $\alpha_{X}(S)=f$, iff and if compute new abstractions that represent $f \wedge\left(\wedge Y_{1} \leftrightarrow \wedge Y_{2}\right)$ and $f \wedge\left(\wedge Y_{1} \leftarrow \wedge Y_{2}\right)$.

Definition 11. The two maps iff : $\wp(X) \times \wp(X) \times$ Share $_{X} \rightarrow$ Share $_{X}$ and if : $\wp(X) \times \wp(X) \times$ Share $_{X} \rightarrow$ Share $_{X}$ are defined by: iff $_{\left(Y_{1}, Y_{2}, S\right)=}$ $\left(S \backslash\left(S_{1} \cup S_{2}\right)\right) \cup\left(S_{1} \otimes S_{2}\right)$ and if $\left(Y_{1}, Y_{2}, S\right)=\left(S \backslash S_{1}\right) \cup\left(S_{1} \otimes S_{2}\right)$ where $S_{1}=\operatorname{rel}\left(Y_{1}, S\right)$ and $S_{2}=\operatorname{rel}\left(Y_{2}, S\right)$.

One important difference between iff and if on the one hand and the abstract unification algorithm of Jacobs and Langen $2: 2$ on the other hand is that iff and if involve no costly closure calculations that arise because of the transitivity of variable sharing. Consequently the complexity iff and if is not exponential in the number of variable sets in $S$, but quadratic. This is a similar efficiency gain to that obtained with the Share pair-sharing quotient of Bagnara et al 3 .

Proposition 5. $\alpha_{X}\left(\right.$ iff $\left.\left(Y_{1}, Y_{2}, S\right)\right)=\alpha_{X}(S) \wedge\left(\wedge Y_{1} \leftrightarrow \wedge Y_{2}\right)$

Corollary 1. $\alpha_{X}\left(\right.$ if $\left.\left(Y_{1}, Y_{2}, S\right)\right)=\alpha_{X}(S) \wedge\left(\wedge Y_{1} \leftarrow \wedge Y_{2}\right)$

Even though if $\left(Y_{1}, Y_{2}, S\right)$ can be simulated with iff $\left(Y_{1}{ }^{\prime}, Y_{2}, S\right)$ where $Y_{1}^{\prime}=Y_{1} \cup$ $Y_{2}$, it is cheaper to compute $\operatorname{rel}\left(Y_{1}, S\right)$ than $\operatorname{rel}\left(Y_{1}{ }^{\prime}, S\right)$. This is one reason why if $\left(Y_{1}, Y_{2}, S\right)$ is more efficient than iff $\left(Y_{1}{ }^{\prime}, Y_{2}, S\right)$. The map if is particularly useful in the analysis of constraint logic programs, where a constraint like $x=y+z$ is abstracted by $(x \leftarrow(y \wedge z)) \wedge(y \leftarrow(x \wedge z)) \wedge(z \leftarrow(x \wedge y))$.

Projection is an important component of a Def analysis within itself 21 . For completeness, we state its correctness as a proposition. 
Definition 12 (projection $\bar{\exists}$ ). The map $\bar{\exists}: \wp(X) \times$ Share $_{X} \rightarrow$ Share $_{X}$ is defined by: $\bar{\exists} Y . S=\{G \cap Y \mid G \in S\} \backslash\{\emptyset\}$.

Proposition 6. If $Y \subseteq X$ then $\exists(X \backslash Y) \cdot \alpha_{X}\left([S]_{\equiv}\right)=\alpha_{Y}\left([\bar{\exists} Y \cdot S]_{\equiv}\right)$.

Finally, Theorem $\amalg$ shows how meet can be computed with a sequence of iff calculations.

Theorem 1. $\left[S_{1}\right]_{\equiv} \sqcap\left[S_{2}\right]_{\equiv}=\left[\bar{\exists} X . S_{n+1}^{\prime}\right]_{\equiv}$ where $X=\left\{x_{1}, \ldots, x_{n}\right\}, S_{1}^{\prime}=\rho\left(S_{1}\right) \cup$ $S_{2}, S_{j+1}^{\prime}=\operatorname{iff}\left(\left\{\rho\left(x_{j}\right)\right\},\left\{x_{j}\right\}, S_{j}^{\prime}\right)$ for $j \in\{1, \ldots, n\}$ and $\rho$ is a renaming such that $\rho(X) \cap X=\emptyset$.

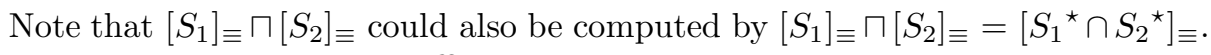
This, however, would be inefficient.

Example 8. Consider calculating $\left[S_{1}\right]_{\equiv} \sqcap\left[S_{2}\right]_{\equiv}$ where $X=\{w, x, y\}, S_{1}=\{\{w, x\}$, $\{x\},\{y\}\}$ and $S_{2}=\{\{w\},\{x, y\},\{y\}\}$. Thus $\alpha_{X}\left(S_{1}\right)=w \leftarrow x$ and $\alpha_{X}\left(S_{2}\right)=$ $x \leftarrow y$. If $\rho=\left\{w \mapsto w^{\prime}, x \mapsto x^{\prime}, y \mapsto y^{\prime}\right\}$ then

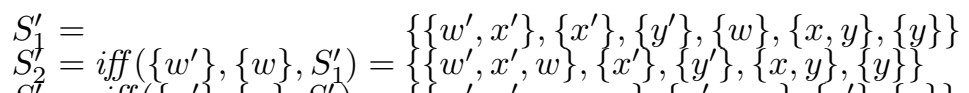

$$
\begin{aligned}
& \left.\left.\begin{array}{l}
S_{3}^{\prime}=\operatorname{iff}\left(\left\{x^{\prime}\right.\right. \\
S_{4}^{\prime}=\operatorname{iff}\left(\left\{y^{\prime}\right.\right.
\end{array}\right\},\{x\}, S_{2}^{\prime}\right)=\left\{\left\{w^{\prime}, x^{\prime}, w, x, y\right\},\left\{x^{\prime}, x, y\right\},\left\{y^{\prime}\right\},\{y\}\right\} \\
& S_{4}^{\prime}=\operatorname{iff}\left(\left\{y^{\prime}\right\},\{y\}, S_{3}^{\prime}\right)=\left\{\left\{w^{\prime}, x^{\prime}, y^{\prime}, w, x, y\right\},\left\{x^{\prime}, y^{\prime}, x, y\right\},\left\{y^{\prime}, y\right\}\right\}
\end{aligned}
$$

Thus $\left[S_{1}\right]_{\equiv} \sqcap\left[S_{2}\right]_{\equiv}=\left[\exists X . S_{4}{ }^{\prime}\right]_{\equiv}=\{\{w, x, y\},\{x, y\},\{y\}\}$. Observe that $\alpha_{X}\left(\left[S_{1}\right]_{\equiv} \sqcap\right.$ $\left.\left[S_{2}\right]_{\equiv}\right)=(w \leftarrow x) \wedge(x \leftarrow y)$ as required.

\section{$5 \quad$ Representing equivalence classes and meet rescheduling}

In our analysis, the functions $f$ and $f^{\prime}$ would be represented by elements of Share $_{X}, S$ and $S^{\prime}$, say. The fixpoint stability check, $f=f^{\prime}$, amounts to checking whether $[S]_{\equiv}=\left[S^{\prime}\right]_{\equiv}$ which, in turn, reduces to deciding whether $\alpha_{X}(S)=$ $\alpha_{X}\left(S^{\prime}\right)$. To make this test efficient we represent an equivalence class by its smallest representative and thus introduce a compression operator $c$.

Definition 13. $c$ : Share $_{X} \rightarrow$ Share $_{X}$ is defined by: $c(S)=\cap\left\{S^{\prime} \mid S^{\prime} \equiv S\right\}$.

The following proposition explains how $c(S)$ is actually computed.

Proposition 7. Let $n=|X|$. Then $c(S)=S_{n}$ where $S_{1}=\{G \in S|| G \mid=1\}$ and $S_{j+1}=S_{j} \cup\left\{G \in S|| G \mid=j+1 \wedge G \notin S_{j}^{\star}\right\}$.

Trivially, if $S \equiv S^{\prime}$, then $c(S)=c\left(S^{\prime}\right)$. From the proposition we also see that $S^{\star}=S_{n}{ }^{\star}=c(S)^{\star}$ and hence if $c(S)=c\left(S^{\prime}\right)$ then $S^{\star}=c(S)^{\star}=c\left(S^{\prime}\right)^{\star}=S^{\prime \star}$ so that $S \equiv S^{\prime}$ by Lemma Hence $c(S)=c\left(S^{\prime}\right)$ iff $S \equiv S^{\prime}$ and thus by testing whether $c(S)=c\left(S^{\prime}\right)$ we can check for the fixpoint condition $S \equiv S^{\prime}$.

When computing $c(S)$ we can test whether $G \notin S_{j}{ }^{\star}$ without actually computing $S_{j}{ }^{\star}$ as follows. Suppose $S_{j}=\left\{G_{1}, \ldots, G_{m}\right\}$ and $G_{0}{ }^{\prime}=G$. Then compute $G_{i}{ }^{\prime}=G_{i-1}{ }^{\prime} \backslash G_{i}$ if $G_{i} \subseteq G$ and put $G_{i}{ }^{\prime}=G_{i-1}{ }^{\prime}$ otherwise. Then $G_{m}{ }^{\prime}=\emptyset$ iff $G \in S_{j}{ }^{\star}$. Using this tactic we can compute $c(S)$ in quadratic time.

Projection can sometimes lead to abstractions that include redundant variable sets as is illustrated below. 
Example 9. Consider $S=\{\{x\},\{y\},\{x, y, z\}\}$ which, incidentally, represents $\alpha_{X}(S)=(z \leftarrow x) \wedge(z \leftarrow y)$. Projecting onto $\{x, y\}$ like so $\bar{\exists}\{x, y\} . S=$ $\{\{x\},\{y\},\{x, y\}\}$ introduces the set $\{x, y\}$, whereas $c(\bar{\exists}\{x, y\} . S)=\{\{x\},\{y\}\}$.

Compression is only applied to check for stability. In our framework, however, projection always precedes a stability check. For example, the answer pattern for a clause is obtained by projecting onto the head variables, and then a stability check is applied to see if other clauses need to be re-evaluated. Thus, in our framework, compression is applied after projection. Compression could be applied more widely though since, in general, iff $\left(Y_{1}, Y_{2}, c(S)\right) \neq c\left(\right.$ iff $\left.\left(Y_{1}, Y_{2}, c(S)\right)\right)$.

Example 10. Let $S=\{\{x\},\{y, x\},\{y, z\},\{x, z\}\}$. Then $c(S)=S$ and iff $(\{y\},\{z\}$, $S)=\{\{x\},\{y, z\},\{y, x, z\}\}$ but $c(\{\{x\},\{y, z\},\{y, x, z\}\})=\{\{x\},\{y, z\}\}$.

In practice, however, the space saving yielded by $c\left(\right.$ iff $\left.\left(Y_{1}, Y_{2}, S\right)\right)$ over iff $\left(Y_{1}, Y_{2}, S\right)$ is usually small and not worth the effort of computing $c$.

Curiously, the efficiency of meet computations can often be significantly improved by introducing some redundancy into the representation. Specifically, a Boolean function is represented by a pair $\langle M, S\rangle$ where $M=X \backslash \operatorname{var}(S)$. The pair $\langle M, S\rangle$ does not include any information that is not present in $S$ : it simply flags those variables, $M$, that are ground (or definite). (This is reminiscent of the reactive ROBDD representation of Bagnara ?.) This is very useful in computing $\left[S_{1}\right]_{\equiv} \sqcap\left[S_{2}\right]_{\equiv}$ by the method prescribed in Theorem II Since meet is

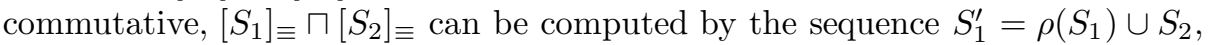
$S_{j+1}^{\prime}=\operatorname{iff}\left(\left\{\rho\left(x_{\pi(j)}\right)\right\},\left\{x_{\pi(j)}\right\}, S_{j}^{\prime}\right)$ where $\pi$ is a permutation on $\{1, \ldots, n\}$. The tactic is to choose a permutation with a maximal $m \in\{0, \ldots, n\}$ such that $\left(\rho\left(x_{\pi(1)}\right) \in M \vee x_{\pi(1)} \in M\right) \ldots\left(\rho\left(x_{\pi(m)}\right) \in M \vee x_{\pi(m)} \in M\right)$ where $M=M_{1} \cup M_{2}$, $M_{1}=X \backslash \operatorname{var}\left(S_{1}\right)$ and $M_{2}=X \backslash \operatorname{var}\left(S_{2}\right)$. We call this technique meet rescheduling, and illustrate its usefulness in the following example.

Example 11. Consider $\left[S_{1}\right]_{\equiv} \sqcap\left[S_{2}\right]_{\equiv}$ where $X=\left\{x_{1}, x_{2}, x_{3}\right\}, S_{1}=\left\{\left\{x_{1}, x_{2}\right\}\right\}$ and $S_{2}=\left\{\left\{x_{1}\right\},\left\{x_{2}, x_{3}\right\}\right\}$. Thus $\alpha_{X}\left(S_{1}\right)=\left(x_{1} \leftrightarrow x_{2}\right) \wedge x_{3}$ and $\alpha_{X}\left(S_{2}\right)=\left(x_{2} \leftrightarrow x_{3}\right)$. Also $M_{1}=\left\{x_{3}\right\}, M_{2}=\emptyset$ and thus $M=\left\{x_{3}\right\}$. If $\rho=\left\{x_{1} \mapsto x_{1}{ }^{\prime}, x_{2} \mapsto x_{2}{ }^{\prime}, x_{3} \mapsto\right.$ $\left.x_{3}{ }^{\prime}\right\}$ then scheduling naively and using $\pi=\{1 \mapsto 3,2 \mapsto 1,3 \mapsto 2\}$ we obtain, respectively

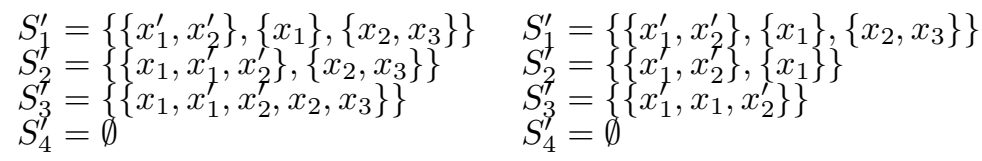

Note how the re-ordering $\pi$ tends to reduce the size of the intermediate $S_{i}^{\prime}$.

A pair $\langle M, S\rangle$ representation is preferred to recomputing $M$ prior to each meet because formulae typically occur as the operands of many meet operations. Thus $M$ serves as a memo, avoiding unnecessary recomputation. 


\section{$6 \quad$ Widening}

Apart from reducing the size of abstractions, it is also worthwhile to avoid generating large abstractions that can arise from the quadratic growth of iff $\left(Y_{1}, Y_{2}, S\right)$ and if $\left(Y_{1}, Y_{2}, S\right)$ stemming from $S_{1} \otimes S_{2}$. However, if $|S|=n,\left|S_{1}\right|=n_{1},\left|S_{2}\right|=n_{2}$ then $\mid$ iff $\left(Y_{1}, Y_{2}, S\right) \mid \leq n+n_{1} n_{2}-\left(n_{1}+n_{2}\right)$. Thus it is possible to detect that $\mid$ iff $\left(Y_{1}, Y_{2}, S\right) \mid$ will definitely be small, say less than a threshold $k$, without computing iff $\left(Y_{1}, Y_{2}, S\right)$ itself. This leads to the following (widened) versions of iff and if that trade precision for efficiency.

\section{Definition 14.}

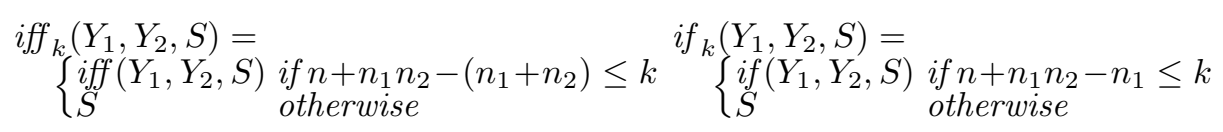

where $S_{1}=\operatorname{rel}\left(Y_{1}, S\right), S_{2}=\operatorname{rel}\left(Y_{2}, S\right),|S|=n,\left|S_{1}\right|=n_{1}$ and $\left|S_{2}\right|=n_{2}$.

This (space) widening ensures that at each stage of the analysis the size of an abstraction is kept smaller than $k$. In fact, since the size of the abstraction depends on the number of variables, $k$ is defined as a multiple of the number of the variables in a clause. This is enough to ensure that, in our interpreter, our space usage grows linearly with the size of the program. A widened meet can be obtained by replacing each iff $\left(\left\{\rho\left(x_{j}\right)\right\},\left\{x_{j}\right\}, S_{j}^{\prime}\right)$ of Theorem II by iff $k\left(\left\{\rho\left(x_{j}\right)\right\},\left\{x_{j}\right\}, S_{j}^{\prime}\right)$. (Interestingly, a widening for ROBDD's is described by Fecht 201 that combats the space problems that arise in the analysis of high arity predicates.)

Folklore 8 says that call and answer patterns rarely get updated more than 3-4 times. This is true for many small programs, but in chat_80.pl and aqua_c.pl we have observed patterns being updated 10-12 times. To bound the number of iterations that can occur, we widen abstractions if they are updated more than, say, 8 times. This (time) widening is defined by: $\triangle(S)=S^{\prime} \cup\{\{x\} \mid x \in \operatorname{var}(S) \backslash$ $\left.\operatorname{var}\left(S^{\prime}\right)\right\}$ where $S^{\prime}=\left\{G^{\prime} \in S \mid \forall G \in S .\left(G \cap G^{\prime} \neq \emptyset\right) \rightarrow\left(G^{\prime} \subseteq G\right)\right\}$. Observe that $[S]_{\equiv} \sqsubseteq[\triangle(S)]_{\equiv}$ and that $\alpha_{X}(\triangle(S))=(\wedge Y) \wedge(\wedge\{x \leftrightarrow y \mid G \in \triangle(S) \wedge x, y \in G\})$ where $Y=X \backslash \operatorname{var}(\triangle(S))$. Formulae of this form occur in the WPos domain of Codish et al 11 and thus have a maximal chain length that is linear in $|X|$. This ensures that the number of iterates will be linear in the sum of the arities of program predicates, and thus provides a time guarantee for a cautious compiler vendor.

\section{Experimental work}

To investigate whether a quadratic meet, meet rescheduling and widening are enough to obtain an efficient and scalable dependency analysis, we have implemented an analyser in Prolog as a simple meta-interpreter that uses induced magic-sets 9 and eager evaluation 27 to perform goal-dependent bottom-up evaluation. Induced magic is a refinement of the magic set transformation, avoiding much of the re-computation that arises because of the repetition of literals in the bodies of magic'ed clauses 9 . It also avoids the overhead of applying the 
magic set transformation. Eager evaluation 27 is a fixpoint iteration strategy which proceeds as follows: whenever an atom is updated with a new (less precise) abstraction, a recursive procedure is invoked to ensure that every clause that has that atom in its body is re-evaluated. Eager evaluation can involve more re-computation than semi-naive iteration but it has the advantages that (1) a $(\Delta$-)set of recently updated atoms does not need to be represented; (2) eager evaluation performs a depth-first traversal of the call-graph so that information about strongly connected components (SCCs) of the call-graph is not as important as in semi-naive iteration. Thus we also avoid computing SCCs.

\begin{tabular}{|c|c|c|c|c|c|c|c|c|c|c|}
\hline file & abs & Con & $D e f_{n}$ & $D e f_{r}$ & $D e f_{w}$ & Pos & & Def & $D e f_{w}$ & Pos \\
\hline disj_r.pI & 0.13 & 0.06 & 0.13 & 0.09 & 0.08 & 0.03 & 38 & 97 & $\frac{j w}{97}$ & 97 \\
\hline scc1.pl & 0.21 & 0.13 & 0.81 & 0.81 & 0.77 & 0.37 & 44 & 89 & 89 & 89 \\
\hline tictactoe.pl & 0.22 & 0.02 & 0.12 & 0.08 & 0.09 & 0.04 & 56 & 56 & 56 & 56 \\
\hline dialog.pl & 0.13 & 0.03 & 0.1 & 0.07 & 0.07 & 0.04 & 46 & 70 & 70 & 70 \\
\hline _v2-2-1.pl & 0.18 & 0.05 & 0.56 & 0.3 & 0.29 & 0.36 & 77 & 101 & 101 & 101 \\
\hline cs_r.pl & 0.26 & 0.06 & 0.11 & 0.1 & 0.09 & 0.05 & 36 & 149 & 149 & 149 \\
\hline flatten.pl & 0.17 & 0.03 & 0.76 & 0.36 & 0.35 & 1.62 & 26 & 27 & 27 & 27 \\
\hline conman.pl & 0.2 & 0.0 & 0.01 & 0.02 & 0.01 & 0.01 & 6 & 6 & 6 & 6 \\
\hline unify.pl & 0.21 & 0.04 & 0.87 & 0.45 & 0.44 & 59.06 & 69 & 70 & 70 & 70 \\
\hline bridge.clpr & 0.34 & 0.01 & 0.06 & 0.03 & 0.03 & 0.04 & 24 & 24 & 24 & 24 \\
\hline neural.clpr & 0.25 & 0.05 & 0.58 & 0.15 & 0.14 & 0.12 & 80 & 118 & 118 & 118 \\
\hline kalah.pl & 0.22 & 0.1 & 0.12 & 0.13 & 0.12 & 0.04 & 91 & 199 & 199 & 199 \\
\hline bryant.pl & 0.28 & 0.06 & 1.46 & 1.04 & 1.03 & 70.24 & 89 & 89 & 89 & 89 \\
\hline nbody.pl & 0.33 & 0.05 & 8.44 & 0.33 & 0.32 & 1.03 & 83 & 109 & 109 & 109 \\
\hline sdda.pl & 0.25 & 0.04 & 0.34 & 0.38 & 0.38 & 3.33 & 17 & 17 & 17 & 17 \\
\hline peep.pl & 0.51 & 0.04 & 0.37 & 0.3 & 0.29 & 0.87 & 8 & 10 & 10 & 10 \\
\hline boyer.pl & 0.32 & 0.03 & 0.11 & 0.18 & 0.19 & 0.15 & 3 & 3 & 3 & 3 \\
\hline read.pl & 0.38 & 0.05 & 0.81 & 0.42 & 0.4 & 1.15 & 90 & 99 & 99 & 99 \\
\hline qplan.pl & 0.36 & 0.12 & 1.77 & 1.59 & 1.56 & 63.8 & 42 & 49 & 49 & 49 \\
\hline reducer.pl & 0.31 & 0.04 & $\infty$ & $\infty$ & 0.93 & $\infty$ & 36 & - & 41 & \\
\hline press.pl & 0.36 & 0.17 & 11.6 & 4.23 & 1.26 & 2.29 & 45 & 53 & 53 & 53 \\
\hline asm.pl & 0.51 & 0.07 & 0.33 & 0.39 & 0.43 & 0.23 & 86 & 87 & 87 & 87 \\
\hline cser_dcg.pl & 0.39 & 0.08 & 0.53 & 0.58 & 0.55 & 0.97 & 25 & 41 & 41 & 41 \\
\hline trs.pl & 0.52 & 0.09 & 3.08 & 2.51 & 2.48 & $\infty$ & 13 & $\begin{array}{l}13 \\
13\end{array}$ & 13 & $\infty$ \\
\hline dbqas.pl & 0.36 & 0.02 & 0.31 & 0.09 & 0.09 & 0.23 & 36 & 43 & 43 & 43 \\
\hline ann.pl & 0.41 & 0.09 & 1.9 & 1.27 & 0.94 & 1.99 & 73 & 73 & 73 & 73 \\
\hline nanc & 0.4 & 0.62 & 0.67 & 0.39 & 0.39 & 0.16 & 123 & 402 & 402 & 402 \\
\hline _analyzer.pl & 0.3 & 0.08 & 2.37 & 0.74 & 0.72 & $\infty$ & 88 & 89 & $8 \overline{9}$ & \\
\hline sim.pl & 0.76 & 0.18 & 3.62 & 2.51 & 2.46 & $\infty$ & 81 & 100 & 100 & - \\
\hline ili.pl & 0.61 & 0.13 & $\infty$ & $\infty$ & 1.69 & 19.16 & 4 & 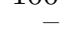 & 4 & 4 \\
\hline Tnprolog.pl & 0.41 & 0.16 & 0.37 & 0.53 & 0.5 & 0.23 & 54 & 143 & 143 & 143 \\
\hline & 0.79 & 0.2 & 2.0 & 1.96 & 1.93 & $\infty$ & 153 & 160 & 160 & \\
\hline & 0.79 & 0.04 & 0.17 & 0.16 & 0.16 & 0.06 & 144 & 144 & 144 & 144 \\
\hline peval.pl & 0.6 & 0.08 & 1.92 & 1.49 & 1.06 & 9.34 & 27 & 27 & 27 & 27 \\
\hline sim_v5-2.pl & $0 . \varepsilon$ & 0.11 & 0.48 & 0.55 & 0.54 & 0.49 & 100 & 101 & 101 & 101 \\
\hline chat_pa & 1.0 & 0.62 & 4.27 & 3.88 & 3.52 & $\infty$ & 444 & 505 & 505 & \\
\hline & & $8:$ & 1.34 & 1.3 & 1.3 & 0.35 & 228 & 687 & 687 & 687 \\
\hline & 1.4 ? & 0.25 & 2.76 & 1.43 & 1.39 & 17.44 & 103 & 155 & 155 & 155 \\
\hline cha & 4.63 & 1.23 & 12.89 & 9.99 & 9.82 & $\infty$ & 457 & 839 & 839 & - \\
\hline & & & $\infty$ & & 69.56 & $\infty$ & 1087 & & 1227 & 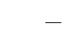 \\
\hline
\end{tabular}

The table summarises our experimental results for applying Def to some of the largest Prolog and $\operatorname{CLP}(\mathcal{R})$ benchmark programs that we could find on the WWW. The programs are ordered by size, where size is measured in terms of the number of (distinct abstract) clauses. To assess the precision of the Def analysis, we have implemented a standard Pos analysis following the technique of Codish and Demoen 10. Ideally our Def analysis should match its precision. We have also modified this analysis to obtain a Con analysis 2.3 . Ideally our Def analysis 
should significantly improve on its precision, since otherwise neither Def or Pos are worthwhile! For completeness, we have included the timings for Pos and Con, but we are primarily concerned with precision. Our Pos analysis is not state-ofthe-art. The abs column give the time for parsing the files and abstracting them, that is, replacing built-ins, like $\arg (\mathrm{X}, \mathrm{T}, \mathrm{S})$, with formulae, like $\mathrm{X} \wedge(\mathrm{S} \leftarrow \mathrm{T})$. This overhead is the same for all the analyses. The fixpoint columns gives the time to compute the fixpoint. $D e f_{n}$ is a naive implementation of our analysis (that took two person weeks to construct) which applies compression but not meet rescheduling and widening; De $f_{r}$ additionally applies meet rescheduling; and $D e f_{w}$ applies compression, meet rescheduling and widening. The $D e f_{r}$ and $D e f_{w}$ analysers were developed together and took an additional 4 days to construct. The code for $D e f_{n}, D e f_{r}$ and $D e f_{w}$ meta-interpreters (including all the set manipulation utilities) is less than 700 clauses. We widen for time at iteration 8 and widen for space when the number of variable sets is more than 16 times the number of variables in a clause. Times are in seconds and $\infty$ indicates that the fixpoint calculation timed out after two minutes. The timings were carried out on an Sun-20 SuperSparc with 64 MByte to match the architecture of Fecht 20. The analysers were coded in SICStus $3 \# 5$ and compiled to naive code. The precision columns give the total number of ground arguments in the call and answer patterns: this is an absolute measure which reflects the usefulness of the analysis for code optimisation. The precision figures for $D e f_{n}$ and $D e f_{r}$ are the same and given in column Def.

The experimental results indicate that $D e f_{w}$ has good scaling behaviour. This is the crucial point. Put simply, there are no programs for which Pos terminates within two minutes and Defw does not (although Pos is sometimes faster). Usually meet rescheduling gives a speedup and sometimes this speedup is very dramatic. $10 \%$ of the programs, however, run slower with meet rescheduling. This typically occurs in programs with very few ground arguments where the effort of rescheduling in not repaid by a reduction in the size of sharing abstractions. Widening seems to be crucial for scalability as is illustrated by reducer, ili and aqua_c. Widening, in fact, is rarely applied. It is crucial for efficiency though because, just one large sharing abstraction can have a disastrous impact on performance. (This also suggests that widening is necessary in the pair-sharing quotient of Share 3 .)

Since our machine matches that of Fecht 20 we can also compare the speed of our Def analyser to the BDD-based Pos GENA analyser 2010. This the one of the fastest (perhaps the fastest) Pos analysis that is described in the literature. With the sophisticated CallWDFS 20 framework, ann.pl takes 0.18 s, nand.pl takes 0.31 s, chat_80.pl takes 4.29 s, and aqua_c.pl takes 28.54 s. Since Fecht 20 does not give processor details for his Sparc-20, we have run our experiments on the slowest $50 \mathrm{MHz}$ model that was manufactured. His machine could well be almost twice as fast. Even though our framework is not semi-naive, we are (at most) 2-4 times as slow as GENA. Furthermore, to perform a comparison against China instantiated with Pos 4 , Bagnara has run $D e f_{n}$ and China on a Pentium 200MHz PC with 64 MByte of memory. On trs.pl and chat_80.pl De $f_{n}$ 
take $3.17 \mathrm{~s}$ and $12.59 \mathrm{~s}$ respectively running interpreted SICStus $3 \# 6$ bytecode. CHINA takes $2.94 \mathrm{~s}$ and $6.24 \mathrm{~s}$ respectively. It seems reasonable to assume that with $D e f_{w}$ on the same PC, trs.pl and chat_80.pl would take $3.17 \times \frac{2.48}{3.08} \approx 2.55 \mathrm{~s}$ and $12.59 \times \frac{9.82}{12.89} \approx 9.59 \mathrm{~s}$. This performance gap for chat_80.pl would be closed if naive code assembly was available for the PC. To summarise, the experimental results are very encouraging and despite the simplicity of the interpreter, our $D e f_{w}$ analysis appears to be fast, precise and scalable and, of course, can be implemented easily in Prolog.

\section{Related work}

Cortesi et al I5 first pointed out that Share expresses the groundness dependencies of Def. Quotienting was introduced by Cortesi et al If as a systematic way of obtaining the reference domain of 15 . Like Bagnara et al 3, we do not fully adhere to the quotienting terminology and methodology of Cortesi et al 15 but rather follow the standard convention $\mathrm{I} 7$ of inducing an equivalence relation (三) from an abstraction map $\left(\alpha_{X}\right)$. Also, Lemma 6.2 of 16 can be interpreted as a way of computing the meet in Def with the classic abstract unification of Jacobs and Langen 22 . We take this further and show how the meet can be computed without exponential time closure operations.

Bagnara et al 3 point out that Share includes redundant sharing information with respect to pair-sharing. This work is related to ours in that our domain may be viewed as a further quotient of the pair-sharing domain. However, widening has not been explored for the pair-sharing domain although, we have shown that even for our simpler domain, that widening is crucial for scalability.

Armstrong et al 1 investigate various normal forms of Boolean functions and the relative precision of Pos and Def. C-based implementations of each representation are described. For the representations of Pos, it is concluded that ROBDD's give the fastest analysis. A specialised representation for Def, based on Dual Blake Canonical Form (DBCF), is found to be the fastest overall. For medium-sized programs it is several times faster than ROBDD's, and it is concluded that this is the representation likely to scale best for real programs. The precision achieved using Pos was found to be significantly higher than Def, although it is remarked that a top-down analyser would improve the precision of Def since it is not condensing. Our findings support this remark.

Bagnara and Schachte 4 develop the idea that a hybrid implementation of ROBDD's that keeps definite information separate from dependency information is more efficient than keeping the two together. This hybrid representation can significantly decrease the size of ROBDD's and thus is a useful implementation tactic. A comparison with our Def analysis has already been given. Fecht 20) compares his Pos analyser to that of Van Hentenryck et al 26 and concludes that his analyser is an order of magnitude faster. For reasons of space, the reader is referred to 20 pp. 305-307] for more details. Performance figures for another hybrid representation are given in 24 . We just observe that 4 and 20 are very good systems to measure against. 
García de la Banda et al 21 represent Def functions in terms of a domain $\wp(X) \times \wp(X \times \wp(\wp(X)))$, so that the Herbrand constraint $x=f(y, z)$, for example, is represented by $\langle\emptyset,\{\langle x,\{\{y, z\}\}\rangle,\langle y,\{\{x\}\}\rangle,\langle z,\{\{x\}\}\rangle\}\rangle$ which encodes $x \leftrightarrow(y \wedge z)$. Abstract conjunction is expressed in terms of six rewrite rules that put conjunctions of formulae into a normal form. Although not stated, the normal form is essentially the (orthogonal) reduced monotonic body form 1 in which a definite function is represented as $f=\wedge_{x \in X}\left(x \leftarrow M_{x}\right)$ where $M_{x} \in \operatorname{Mon}_{X}$ and $x \notin M_{x}$. Orthogonality ensures that the meet is safe. Our work shows how this symbolic manipulation of definite function can be replaced with a simpler domain and simpler join and meet operations.

Corsini et al 12 describe how variants of Pos can be implemented using Toupie, a constraint language based on the $\mu$-calculus. This BDD-based analysis appears to be at least five times as fast as 26 for success pattern analysis. Thus, if the analyser was extended with magic sets, say, it might lead to a very respectable goal-dependent analysis.

Codish and Demoen 10 describe a truth-table based implementation technique for Pos that would encode $\left(x_{1} \leftrightarrow\left(x_{2} \wedge x_{3}\right)\right)$ as three tuples $\langle$ true, true, true $\rangle$, $\langle$ false, _, false $\rangle$, 〈false, false,__. A widening for this Pos analysis, WPos, is proposed by Codish et al II that amounts to a sub-domain of Def that cannot propagate dependencies of the form $y \leftrightarrow(y \wedge z)$, but only simple dependencies like $(x \leftrightarrow y)$. The main finding of Codish et al II is that WPos looses only a small amount of precision for goal-dependent analysis of Prolog and $\operatorname{CLP}(\mathcal{R})$ programs.

\section{Conclusions}

We have developed the link between Def and Share to show how the meet of Def can be modelled with an efficient (quadratic) operation on Share. We have shown how to represent formulae succinctly with equivalence classes of sharing abstractions, and how formulae can be widened so as to avoid bad space behaviour. Putting these ideas together we have achieved a practical analysis that is fast, precise, robust and can be implemented easily in Prolog.

\section{References}

1. T. Armstrong, K. Marriott, P. Schachte, and H. Søndergaard. Two Classes of Boolean Functions for Dependency analysis. Science of Computer Programming, 31(1):3-45, 1998.

2. R. Bagnara. A Reactive Implementation of Pos using ROBDDs. In Programming Languages: Implementation, Logics and Programs, pages 107-121. Springer-Verlag, 1996. LNCS 1140.

3. R. Bagnara, P. Hill, and E. Zaffanella. Set-Sharing is Redundant for Pair-Sharing. In Static Analysis Symposium, pages 53-67. Springer-Verlag, 1997. LNCS 1302.

4. R. Bagnara and P. Schachte. Factorizing Equivalent Variable Pairs in ROBDDBased Implementations of Pos. In Seventh International Conference on Algebraic Methodology and Software Technology, Amazonia, Brazil, 1999. Springer-Verlag.

5. N. Baker and H. Søndergaard. Definiteness analysis for $\operatorname{CLP}(\mathcal{R})$. In Australian Computer Science Conference, pages 321-332, 1993. 
6. P. Bigot, S. Debray, and K. Marriott. Understanding finiteness analysis using abstract interpretation. In Joint International Conference and Symposium on Logic Programming, pages 735-749. MIT Press, 1992.

7. R. Bryant. Symbolic Boolean manipulation with ordered binary-decision digrams. ACM Computing Surveys, 24(3):293-318, 1992.

8. M. Codish. Worst Case Groundness Analysis. Technical report, Ben-Gurion University of the Negev, 1998. ftp://ftp.cs.bgu.ac.il/pub/people/mcodish/ pathpos.ps.gz.

9. M. Codish. Efficient Goal Directed Bottom-up Evaluation of Logic Programs. J. of Logic Programming (to appear), 1999.

10. M. Codish and B. Demoen. Analysing Logic Programs using "prop"-ositional Logic Programs and a Magic Wand. J. Logic Programming, 25(3):249-274, 1995.

11. M. Codish, A. Heaton, A. King, M. Abo-Zaed, and P. Hill. Widening Positive Boolean functions for Goal-dependent Groundness Analysis. Technical Report 1298, Computing Laboratory, May 1998. http://www.cs.ukc.ac.uk/pubs/1998/589.

12. M.-M. Corsini, K. Musumbu, A. Rauzy, and B. Le Charlier. Efficient Bottomup Abstract Interpretation of Prolog by means of Constraint Solving over Finite Domains. In In proceedings of Programming Language Implementation and Logic Programming, pages 75-91. Springer-Verlag, 1993. LNCS 714.

13. A. Cortesi and G. Filé. Comparison and Design of Abstract Domains for Sharing Analysis. In Italian Conference on Logic Programming, pages 251-265, 1993.

14. A. Cortesi, G. Filé, R. Giacobazzi, C. Palamidessi, and F. Ranzato. Complementation in Abstract Interpretation. ACM TOPLAS, 19(1):7-47, January 1997.

15. A. Cortesi, G. Filé, and W. Winsborough. Comparison of Abstract Interpretations. In International Conference on Automata, Languages and Programming, pages 521-532. Springer-Verlag, 1992. LNCS 623.

16. A. Cortesi, G. Filé, and W. Winsborough. The Quotient of an Abstract Interpretation for Comparing Static Analyses. Theoretical Computer Science, 202(1-2):163192, 1998.

17. P. Cousot and R. Cousot. Abstract interpretation: A unified lattice model for static analysis of programs by construction or approximation of fixpoints. In Principles of Programming Languages, pages 238-252. ACM Press, 1977.

18. P. Dart. On Derived Dependencies and Connected Databases. J. Logic Programming, 11(1\&2):163-188, 1991.

19. S. Dawson, C. R. Ramakrishnan, and D. S. Warren. Practical Program Analysis Using General Purpose Logic Programming Systems - A Case Study. In Programming Language Design and Implementation, pages 117-126. ACM Press, 1996.

20. C. Fecht. Abstrakte Interpretation logischer Programme: Theorie, Implementierung, Generierung. $\mathrm{PhD}$ thesis, Universität des Saarlandes, 1997. http://www.cs.uni-sb.de/RW/users/fecht/.

21. M. García de la Banda, M. Hermenegildo, M. Bruynooghe, V. Dumortier, G. Janssens, and W. Simoens. Global Analysis of Constraint Logic Programs. ACM TOPLAS, 18(5):564-614, 1996.

22. D. Jacobs and A. Langen. Static Analysis of Logic Programs. J. Logic Programming, 13(2 and 3):154-314, 1992.

23. N. Jones and H. Søndergaard. A semantics-based framework for the abstract interpretation of Prolog. In Abstract Interpretation of Declarative Languages, pages 123-142. Ellis Horwood Ltd, 1987.

24. P. Schachte. Precise and Efficient Static Analysis of Logic Programs. PhD thesis, Department of Computer Science, The University of Melbourne, Melbourne, Australia, 1998. (to appear).

25. H. Seidel. Personal communication on Prolog compiler integration and SML. November 1997.

26. P. Van Hentenryck, A. Cortesi, and B. Le Charlier. Evaluation of the domain Prop. J. Logic Programming, 23(3):237-278, 1995.

27. J. Wunderwald. Memoing Evaluation by Source-to-Source Transformation. In Logic Program Synthesis and Transformation, pages 17-32. Springer, 1995. LNCS 1048. 